Physics Vol. 1. No. 4, pp. 209-213, $1965 . \quad$ Physics Publishing Co. Printed in Great Britain.

\title{
CLASSICAL EXAMPLES OF SPACE INVERSION AND TIME REVERSAL*
}

\author{
L. I. SCHIFF \\ Institute of Theoretical Physics, Department of Physics, \\ Stanford University, Stanford, California \\ (Received 18 December 1964 )
}

\begin{abstract}
The motions of four different classical systems under space inversion $(P)$ and time reversal $(T)$ are discussed. It is imagined that each system is photographed with a motion picture camera, and that the film is developed and positively printed. The film may then be inverted front for back and run through the projector in normal time sequence $(P)$, or run through the projector in the correct orientation but backward in time $(T)$. If the projected image represents a possible motion of the system, we say that it is $P$ or $T$ invariant, as the case may be. Classical models of a moving charge, a precessing magnetic dipole, a moving magnetic monopole, and a precessing electric dipole, are considered. As might be expected, the first two systems turn out to be separately $P$ and $T$ invariant, and the last two are neither $P$ nor $T$ invariant. A brief comment is made on charge conjugation $(C)$ invariance and the classical validity of CPT invariance.
\end{abstract}

IT has long been recognized that a system in a nondegenerate quantum state cannot posses a static electric dipole moment if the Hamiltonian is invariant with respect to space inversion $(P)$. Shortly after the suggestion of Lee and Yang [1] that weak interactions are not $P$ invariant, Landau [2] pointed out that invariance under the combined operation $C P$ of charge conjugation $(G)$ and space inversion is needed to rule out the existence of static electric dipole moments of elementary particles. It also follows from the presumed validity of the CPT theorem that $C P$ invariance implies invariance under time reversal $(T)$.

The present paper shows how $P$ and $T$ can be described in simple classical terms, and in particular related to the existence of electric dipole moments. It is imagined that a system of moving objects is photographed with a motion picture camera, and that the film is developed and positively printed. In order to study the $P$ invariance of the system, the film is inverted front for back and run through the projector in normal time sequence. If the projected image represents a possible motion of the system, we say that it is invariant with respect to space inversion. In similar fashion, the film may be run through the projector in the correct orientation, but backward in time. If this projected image represents a possible motion of the system,

* Supported in part by the U.S. Air Force through Air Force Office of Scientific Research Contract AF $49(638)-1389$.

+ A portion of this paper was presented at the New York meeting of the American Association of Physics Teachers, January 1964; see American Journal of Physics 32.812 (1964). 
we say that it is invariant with respect to time reversal. Charge conjugation will be discussed briefly at the end of the paper.

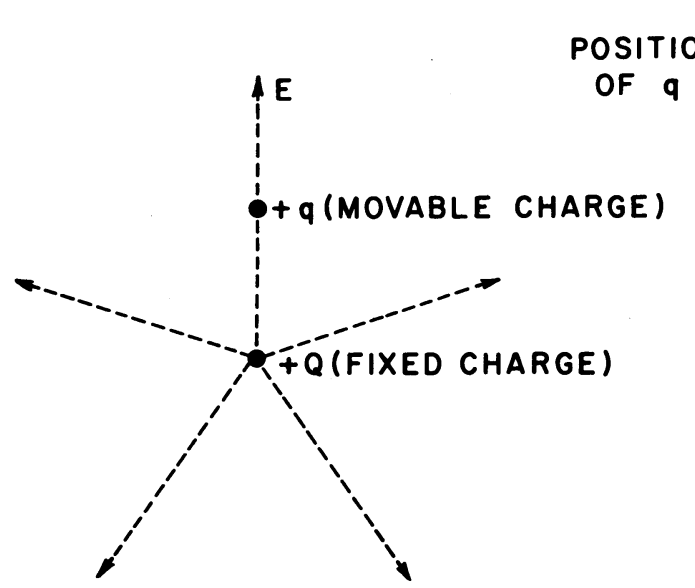

(a)

FIGURE 1

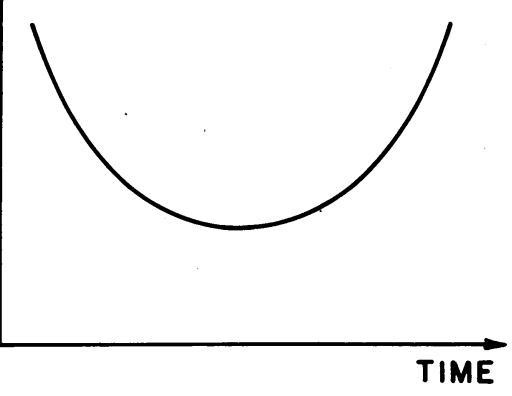

Motion of massive charge $q$ under the influence of a fixed charge $Q$.

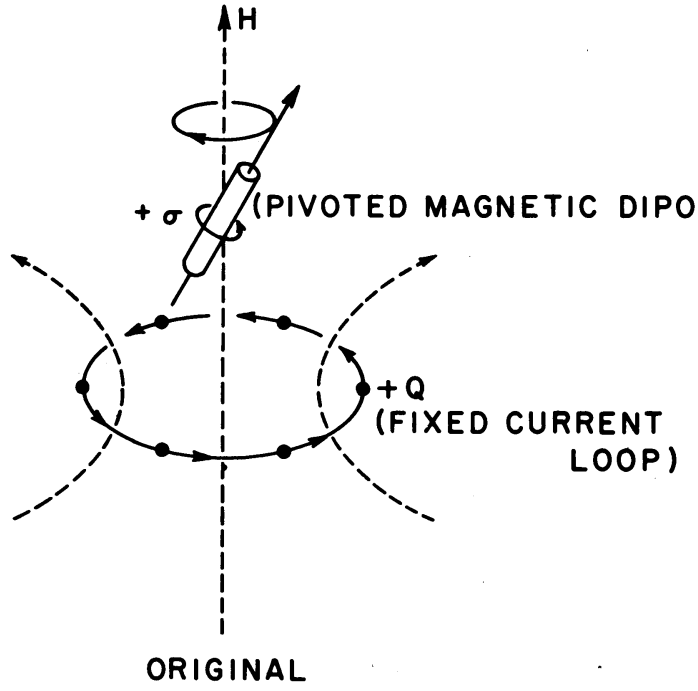

(a)

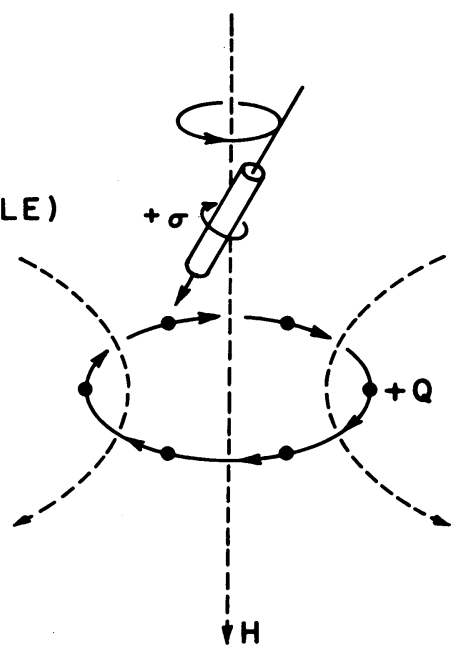

REVERSED

(b)

FIGURE 2

(a) Precession of a magnetic dipole pivoted at a fixed point under the influence of a fixed current loop.

(b) Motion of the system illustrated in (a) when reversed in $t$ ime.

We consider four different systems: a particle of finite mass and charge that moves under the influence of a fixed charge; a massive rotating charged cylinder (magnetic dipole) that is on a fixed pivot and precesses under the influence of a fixed current loop; a particle of finite mass that carries a magnetic monopole and moves under the influence of a fixed current loop; 
and a massive rotating cylinder with an electric dipole moment proportional to its angular momentum, that is on a fixed pivot and precesses under the influence of a fixed charge. As might be expected, the first two systems turn out to be separately $P$ and $T$ invariant, and the last two are neither $P$ nor $T$ invariant.

These four systems are illustrated in Figs. 1 through 4 , respectively. In each case the observable objects and their motions are represented by solid circles and lines. On the other hand, electric and magnetic fields, which are useful constructs for determining the motions but otherwise not directly observable, are represented by dashed lines.

Figure 1(a) shows a particle of finite mass and positive charge $q$ that is free to move under the influence of a fixed positive charge $Q$. Figure $1(b)$ is a schematic plot of the vertical position of $q$ against time, when the particle is initially aimed directly at $Q$. An easy way in which to visualize the space-inverted system is to imagine that Fig. $1(a)$ is printed on transparent paper and is viewed through the back. It is apparent that this changes neither the appearance of the system shown in Fig. 1(a) nor the development of the system in time shown in Fig. 1(b). Thus the space-inverted motion is a possible one for the system so that it is, according to our definition, $P$ invariant. Owing to the symetry of the curve of Fig. $1(b)$ about its lowest point, the system is also $T$ invariant.

Figure 2(a) shows a classical magnetic dipole, which consists of a massive cylinder with positive surface charge density $\sigma$, rotating as shown. Its angular momentum vector and magnetic dipole moment both point in the direction of the arrow drawn along its axis. The center of the dipole is pivoted at a fixed point, and it is free to precess under the influence of a fixed current loop. This loop consists of positive charges $Q$ moving in a circular path. The dashed magnetic field lines $H$ are drawn for the convenience of the reader, and do not constitute a directly observable part of the system. The dipole precesses in the direction shown.

Figure 2(b) shows the appearance of the system when the film is run backward through the projector. The direction of rotation of the cylinder is reversed, as are its angular momentum and magnetic dipole moment. The direction of motion of the charges in the current loop is reversed, so that the magnetic field believed to be present is also reversed. Finally, the direction of precession is reversed. It is apparent that Fig. 2(b) represents a possible motion, so we say that the system is $T$ invariant. If Fig. $2(a)$ is viewed through the back of the paper, it is essentially the same as Fig. $2(b)$. Thus the system is also $P$ invariant.

Figure $3(a)$ shows a particle of finite mass that carries a positive magnetic monopole $m$ and is free to move under the influence of a fixed current loop. Figure $3(b)$ is a schematic plot of the vertical position of $m$ against time, when the particle is projected downward along the axis of the loop. The particle is accelerated upward, and moves as shown if its initial speed is not too great. If now the system is either viewed through the back of the paper or reversed in time, the only change in Fig. $3(a)$ is in the direction of motion of the charges in the current loop. The course of the motion is clearly unchanged by the space inversion, and owing to the symmetry of Fig. $3(b)$ about its lowest point it is not changed by the time reversal either. However, Fig. $3(b)$ no longer represents a possible motion of the system if the current loop is reversed. In this case the particle would be accelerated downward, and its positiontime plot would extend down indefinitely. We conclude that this system is neither $P$ nor $T$ invariant.

There are several physical situations which might be simulated in constructing a classical model of an electric dipole moment. First, there are the excited states of a hydrogen atom which, because of $l$ degeneracy, possess static electric dipole moments that have well-defined components along an externally applied electric field but not in other directions. Second, there are unsymmetrical molecules such as hydrogen chloride, which has a static electric dipole moment that is oriented along the figure axis of the molecule. Third, there may be nuclei 
or elementary particles that possess static electric dipole moments [3]. If so, we assume that the only direction in space with which this moment can be associated is that of the spin axis. The first two situations actually occur in nature as a consequence of electromagnetic interactions that are known to be $P$ and $T$ invariant. Thus we shall consider here only the third situation, and assume that our classical electric dipole is so constructed that rotation generates in it a moment that is directed along its angular momentum.

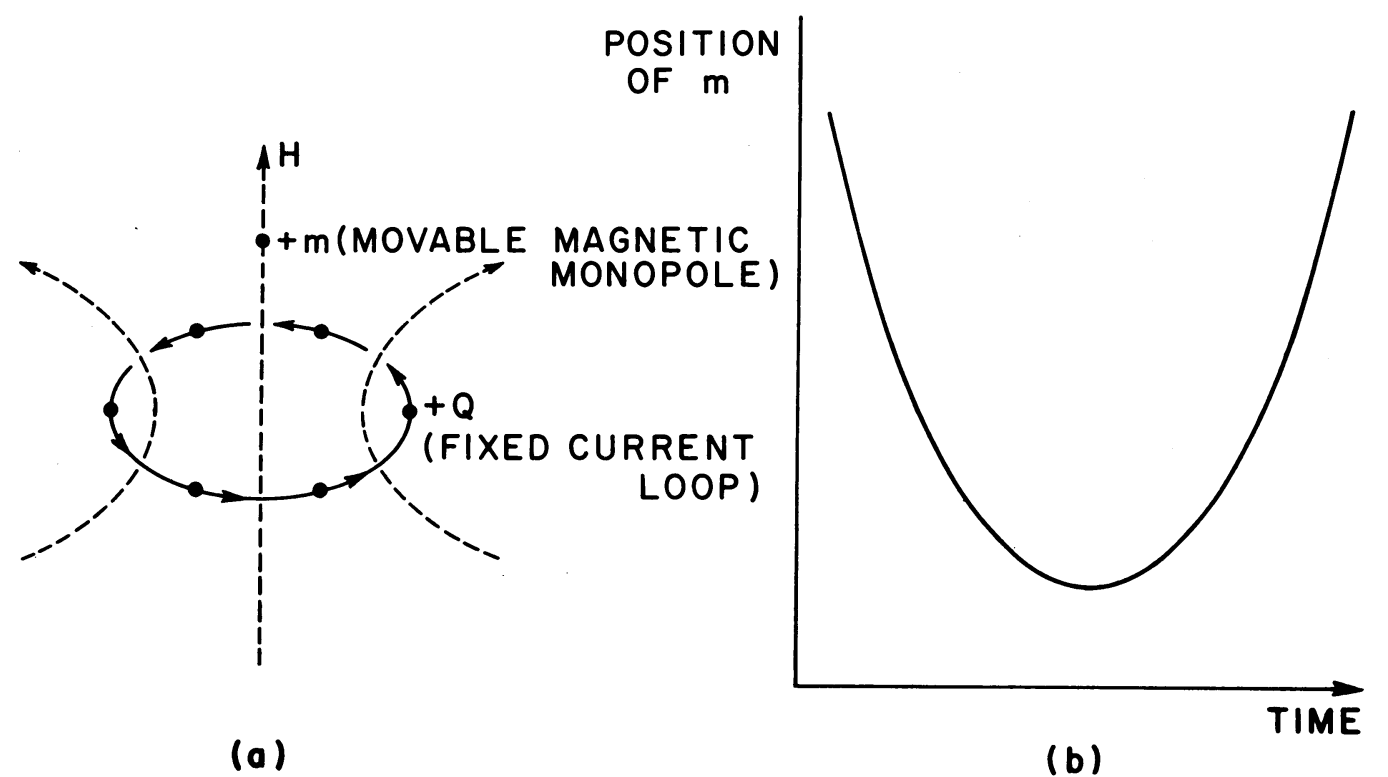

FIGURE 3

Motion of a massive magnetic monopole $m$ under the influence of a fixed current loop.

Figure $4(a)$ shows such a dipole, which consists of a massive rotating cylinder that has a positive charge $q$ on one end and a charge $-q$ on the other, when its angular momentum has a certain magnitude and the direction indicated by the arrow. The center of the dipole is pivoted at a fixed point; it is free to precess under the influence of a fixed positive charge $Q$, and the direction of the precession is as shown. We do not regard the charges $\pm q$ as being directly observable, any more than we regard the electric field $E$ as being directly observable. Rather we infer, on the basis of the observed precession, that rotation is associated with charge separation in the manner shown.

Figure $4(b)$ shows the system as it looks when the film is run backward through the projector; the appearance is essentially the same if Fig. $4(a)$ is viewed through the back of the paper. Again, the charges $\pm q$ marked on the diagram are not directly observable; but now they are inferred from the observed sense of rotation of the cylinder, on the basis of experience with the original system shown in Fig. 4(a). It is apparent, then, that Fig. $4(b)$ does not represent a possible motion of the system. Thus we conclude that this system is neither $P$ nor $T$ invariant.

A classical description of charge conjugation seems to be somewhat more ambiguous than those given above for $P$ and $T$. Perhaps the simplest assumption is that $C$ transformation changes the signs of all charges, magnetic monopoles, magnetic dipole moments, and electric dipole moments. We could then say that one of our systems is $C$ invariant if, after $C$ transformation, it moves in the same way as shown on the left side of the appropriate figure. With this definition, it is apparent that all four systems are $C$ invariant. Furthermore, it is easily seen that they are all $C P T$ invariant, and there is also agreement with Landau's remark that $C P$ invariance is 
incompatible with the existence of elementary static electric dipole moments.

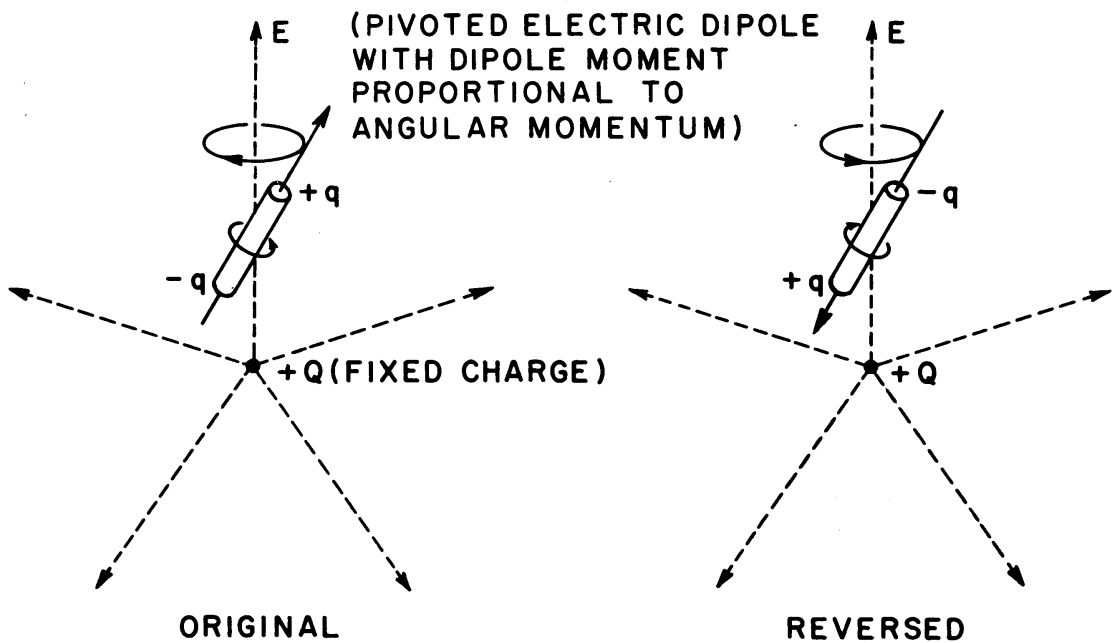

(a)

(b)

FIGURE 4

(a) Precession of an electric dipole that is proportional to its angular momentum under the influence of a fixed charge.

(b) Motion of the system illustrated in $(a)$ when reversed in time.

As a final remark, we note that our conclusions as to which systems do and which systems do not possess $P$ and $T$ invariance derive directly from our assumption as to how electric and magnetic fields are produced. If we had assumed that the magnetic fields in Figs. 2 and 3 are produced by fixed magnetic monopoles and the electric fields in Figs. 1 and 4 by fixed current loops of magnetic monopoles, the conclusions would have been reversed.

NOTE: Dr. P. W. Anderson has remarked that this is equivalent to interchange of what is meant by "electric" and "magnetic", and hence is without physical meaning.

\section{References}

1. T. D. LEE and C.N. YANG, Phys. Rev. 104, 254 (1956).

2. L. LANDAU, Nucl. Phys. 3, 127 (1957).

3. For a discussion of the experimental possibilities, see L.I. SCHIFF, Phys. Rev. 132, 2194 (1963). 